\title{
PCR-DGGE for the profiling of cheese bacterial communities: strengths and weaknesses of a poorly explored combined approach
}

\author{
Lucia Aquilanti $^{1} \cdot$ Sara Santarelli $^{1}$. \\ Valentina Babini $^{1}$ - Andrea Osimani ${ }^{1}$ (D) \\ Cristiana Garofalo $^{1}$ - Serena Polverigiani ${ }^{1}$. \\ Francesca Clementi ${ }^{1}$
}

Received: 7 December 2015 / Revised: 1 June 2016 / Accepted: 2 June 2016 /

Published online: 20 June 2016

(C) INRA and Springer-Verlag France 2016

\begin{abstract}
Polymerase chain reaction-denaturing gradient gel electrophoresis (PCR-DGGE) represents a still valid molecular tool for the profiling of complex microbial ecosystems, including cheeses. In the present study, a double PCRDGGE approach has been applied to the investigation of the bacterial diversity of seven cheese models to objectively assess strengths and weaknesses of such an approach. To that end, the bacterial DNA was extracted directly from both the cheese replicates and the bulks of colonies harvested from the serial dilution agar plates of selective solid media used for the enumeration of presumptive lactobacilli, lactococci and thermophilic cocci, respectively. The results overall collected allowed the main bacterial taxa to be identified and roughly quantified. Rough quantification of the main cultivable species represents a strength of the PCR-DGGE approach applied, whereas its main weaknesses were represented by the low degree of selectivity of the conventional growth media used for cultivation of lactic acid bacteria and the underestimation of the effective microbial diversity occurring in the seven cheese models.
\end{abstract}

Keywords Direct PCR-DGGE approach · Bulk PCR-DGGE approach · Cheese microbiota $\cdot$ Lactic acid bacteria

Electronic supplementary material The online version of this article (doi:10.1007/s13594-016-0296-z) contains supplementary material, which is available to authorized users.

Andrea Osimani

a.osimani@univpm.it

1 Dipartimento di Scienze Agrarie, Alimentari, ed Ambientali, Università Politecnica delle Marche, via Brecce Bianche, 60131 Ancona, Italy 


\section{Introduction}

Since its introduction into microbial ecology in the early 1990s, denaturing gradient gel electrophoresis (DGGE) of amplified ribosomal (rRNA)-encoding genes has become one of the most widely exploited molecular methods for the investigation of the microbial diversity of food ecosystems, including cheeses (Quigley et al. 2011). In the last decade, the introduction of newly developed sequencing strategies, commonly referred to as next-generation DNA sequencing techniques, has substantially revolutionised the study of genomics and molecular biology, being also successfully applied in food microbiology (Mayo et al. 2014). However, the recent few studies exploiting and comparing high-throughput sequencing and PCR-DGGE surprisingly demonstrated how the latter technique still represents a valid tool for the profiling of complex food microbial communities (Delgado et al. 2013; Garofalo et al. 2015).

To date, two PCR-DGGE approaches have been used by food microbiologists for this specific purpose: a culture-independent approach, hereafter referred to as the "direct approach", which is based on the analysis of the nucleic acids (DNA or RNA) extracted directly from the food matrices, and a culture-based approach, hereafter referred to as the "bulk approach", which by contrast relies on the analysis of the DNA extracted from the bulks of colonies harvested from the dilution agar plates used for counting live microorganisms. The main technical aspects of these two alternative approaches have already been elucidated (Ercolini 2004).

Notwithstanding the great potential of a combined use of the direct and bulk approaches for the monitoring of bacterial community structure in milk-based products, as revealed by a pioneer study conducted by Ercolini et al. (2001), to date this double approach has only sporadically been applied to dairy microbiology, where the "standard" is unquestionably represented by the culture-independent PCR-DGGE analysis.

In the early investigation by Ercolini et al. (2001), the DNA was extracted directly from natural whey cultures for the production of water buffalo Mozzarella cheese and the bulks of colonies harvested from all the serial dilution agar plates (from -1 to the last) of a pool of selective solid media; the DNA was then subjected to PCR-DGGE analysis for the amplification and separation of variable portions of the $16 \mathrm{~S}$ rRNA gene. In the following years, the double PCR-DGGE approach was applied in a few investigations aimed at identifying the microbial diversity of raw milk cheeses, namely, Stilton (Ercolini et al. 2003), water buffalo Mozzarella (Ercolini et al. 2004), Goudatype cheeses (Van Hoorde et al. 2008, 2010), an artisanal fresh sheep cheese produced in a limited area of eastern Croatia (Pogačić et al. 2011) and traditional Italian semihard cheeses (Aquilanti et al. 2013; Ricciardi et al. 2014). However, in all these investigations, the bulk approach consisted in the sole analysis of a few selected plates, namely, the countable plates (Ercolini et al. 2003, 2004; Pogačić et al. 2011), the plates inoculated with the sole lowest dilutions (-1) (Van Hoorde et al. 2008) and the plates inoculated with both the lowest and the highest dilutions (Aquilanti et al. 2013; Ricciardi et al. 2014).

In the current study, the potential of the direct and bulk PCR-DGGE approach originally proposed by Ercolini et al. (2001) for the profiling of dairy microbial communities was explored. To this end, seven semi-hard raw and pasteurised milk cheeses manufactured according to different cheese-making technologies were used. The DNA was extracted directly from three cheese replicates of each cheese type and the bulks of colonies harvested 
from the whole set of dilution agar plates (from -1 to the last) used for the enumeration of presumptive lactobacilli, lactococci and thermophilic cocci, respectively; the cheese and bulk DNA were subsequently subjected to PCR-DGGE analysis of the V6-V8 regions of the 16S rRNA gene for the identification of dominant and minority eubacterial components. The results overall collected allowed main strengths and weaknesses of the combined PCRDGGE approach to be pointed out on an objective basis.

\section{Materials and methods}

\subsection{Bacterial reference strains}

Three reference strains were used for the construction of a DGGE ladder: Lactobacillus brevis NCIMB 8664, Lactococcus lactis subsp. lactis DSM 20729 and Streptococcus thermophilus DSM 20617. These were obtained from the "National Collection of Industrial Marine and Food Bacteria" (http:/www.ncimb.com/culture.html) (NCIMB, Aberdeen, Scotland, UK) and the "Deutsche Sammlung von Mikrorganismen und Zellkulturen” (http://www.dsmz.de/) (DSM, Braunsweig, Germany).

\subsection{Cheese samples}

Seven semi-hard cheeses, including two pecorino (labelled "pe"), two caprino (labelled "cp") and three caciotta (labelled "ca") cheeses were analysed (Table 1). Raw milk cheeses (labelled " $r$ ") were supplied by four artisan dairy producers, while pasteurised milk cheeses (labelled "p") were purchased at retail level from supermarkets in the Ancona area (central Italy). The ripening time, cheese milk composition and type of starter culture used are reported in Table 1. For each semi-hard cheese under study, three cheese wheels produced from the same milk batch and ripened under the same conditions (hereafter referred to as cheese production replicates) were analysed. For each cheese production replicate, a 3-cm-thick section from the cheese core and a 3-cmthick section from the subsurface taken about $2 \mathrm{~cm}$ under the rind were collected and brought together as a unique sample.

\subsection{Culturable cell counting}

One aliquot $(10 \mathrm{~g})$ of each cheese sample was accurately homogenised in $90 \mathrm{~mL}$ of sterile aqueous citrate $2 \%(w / v)$ solution with a Stomacher apparatus (400 Circulator, International PBI, Milan, Italy) for $3 \mathrm{~min}$ at $260 \mathrm{rpm}$. A set of serial dilutions was prepared using the same diluent, and an aliquot $(100 \mu \mathrm{L})$ of each dilution was inoculated and spread in duplicate onto selective solid media added with $250 \mathrm{~mL} . \mathrm{L}^{-1}$ cycloheximide for enumeration. Presumptive lactobacilli were counted on MRS agar (Oxoid, Basingstoke, UK) incubated at $37{ }^{\circ} \mathrm{C}$ for $48 \mathrm{~h}$ under anaerobiosis using anaerobic gas generating sachets (Oxoid AnaeroGen 2.5-L Sachet), whereas presumptive lactococci and thermophilic cocci were counted on M17 agar (Oxoid) incubated at $22{ }^{\circ} \mathrm{C}$ for $48 \mathrm{~h}$ and at $45^{\circ} \mathrm{C}$ for $24 \mathrm{~h}$, respectively (hereafter referred to as

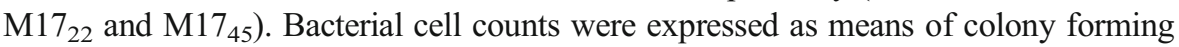
units (cfu) per gram of sample \pm standard deviations. 


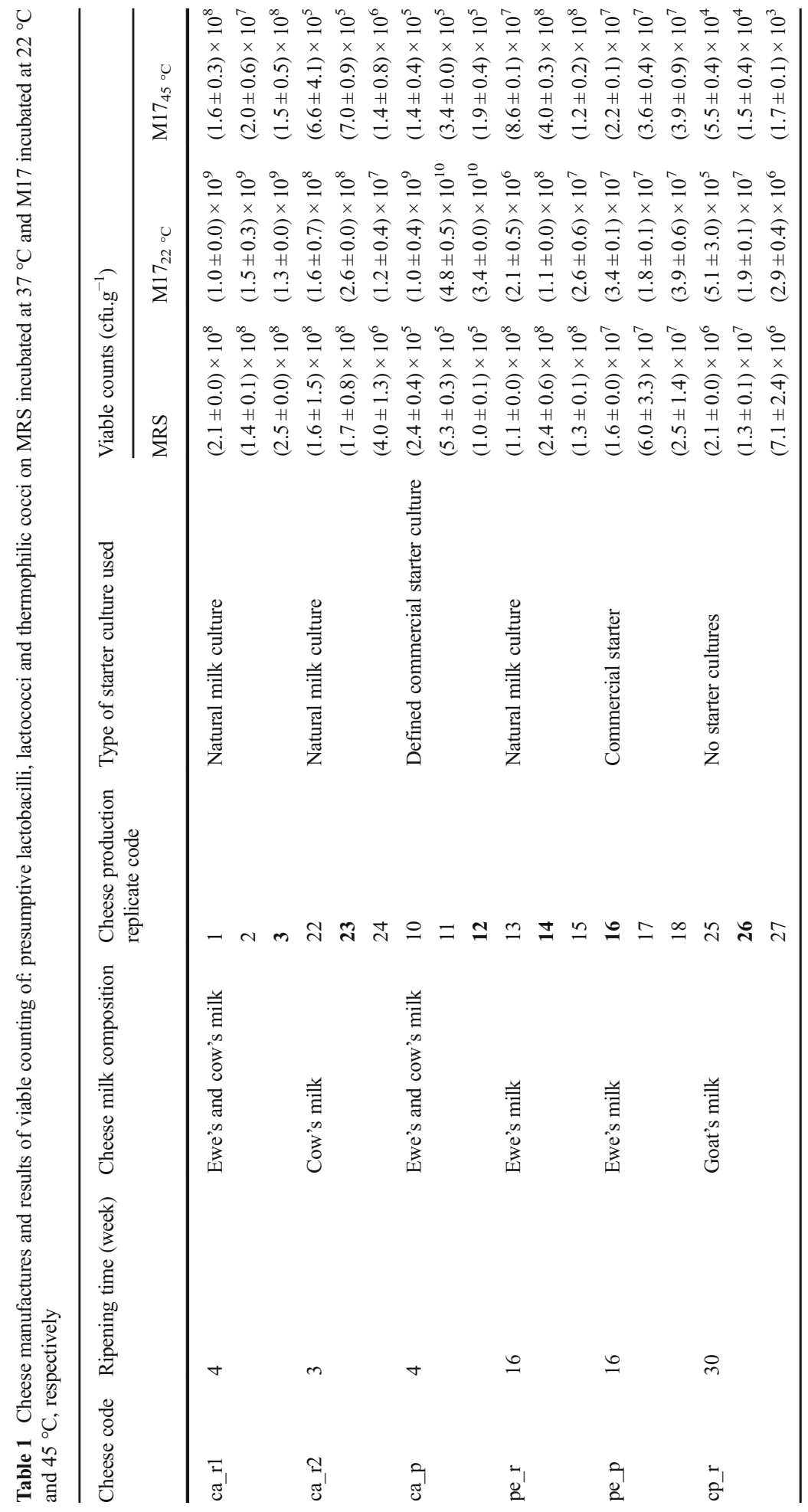




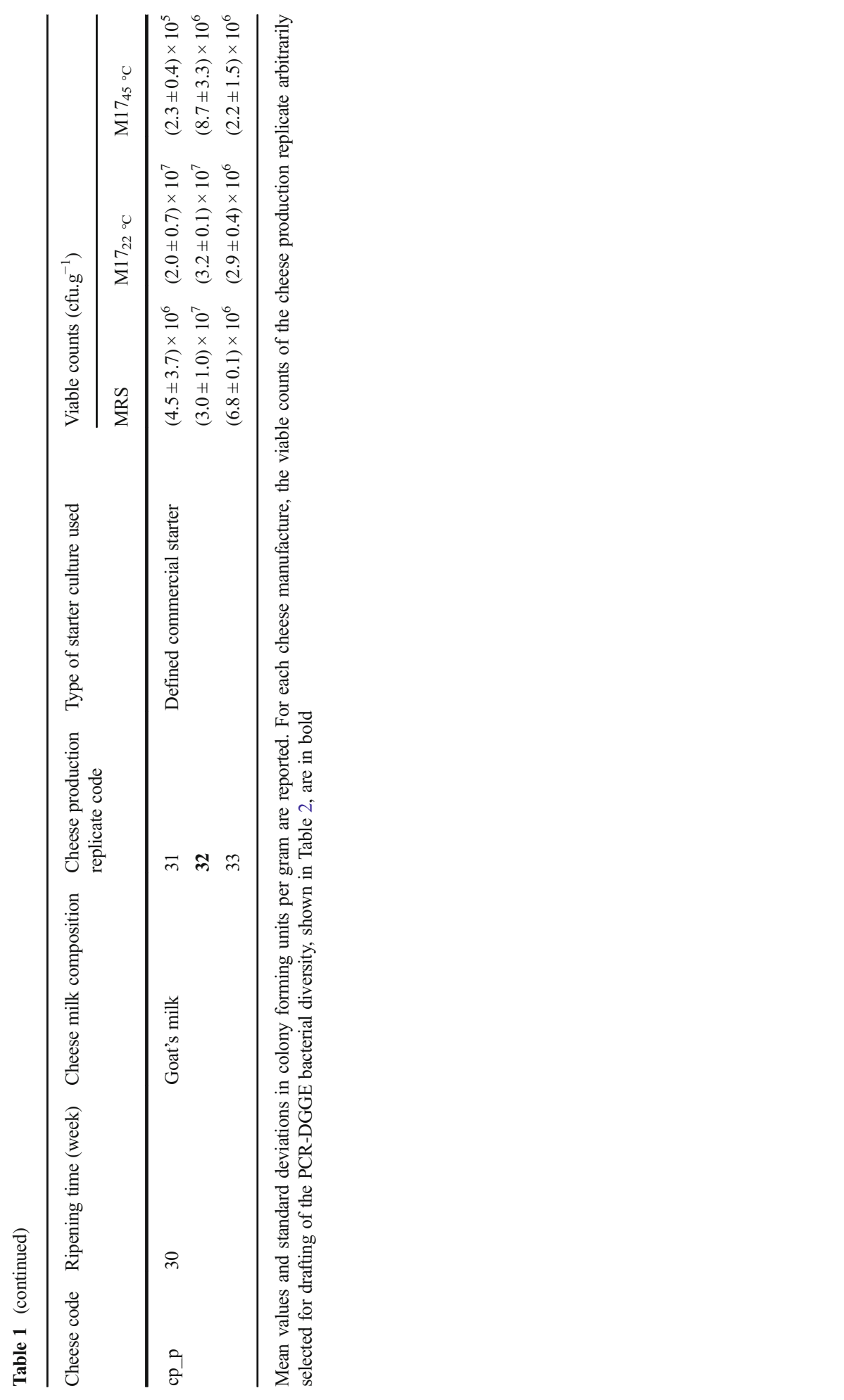




\subsection{DNA extraction from cheese samples and bulks}

The first step in the DNA extraction from the cheese samples was performed as described by Rantsiou et al. (2008), with slight modifications. Briefly, $2 \mathrm{~mL}$ of each cheese homogenate was transferred into a sterile 2-mL tube and centrifuged at $14.000 \times g$ for $10 \mathrm{~min}$. After centrifugation, the fat layer at the top was removed with a sterile cotton tip and the supernatant was eliminated. For the following steps, the microbial DNA extraction procedure described by Osimani et al. (2015) was used. DNA extraction from bulks was carried out following the procedure reported by Aquilanti et al. (2013), modified as follows: for each set of serial dilution agar plates (from -2 to the last), $1.5 \mathrm{~mL}$ of a sterile aqueous $1 \%(w / v)$ peptone solution was poured into plates showing at least five colonies, and the colonies were detached from the agar surface using a disposable L-shaped cell spreader. The resulting bulk suspensions were harvested with a sterile pipette and transferred into a 2-mL tube. The turbidity of DNA extracts from bulk cells was standardised at $1 \mathrm{McFarland}$ unit, to minimise the effect of different concentrations on the biomass of the bulks. The cell pellets were collected by centrifugation at $14.000 \times g$ for $3 \mathrm{~min}$ and washed twice with $1 \mathrm{~mL}$ of sterile deionised water. DNA was extracted from $300 \mu \mathrm{L}$ of each bulk suspension, following the procedure described by Osimani et al. (2015). DNA suspensions obtained with both the direct and the bulk approach were subjected to optical readings at 260, 280 and $234 \mathrm{~nm}$ with a UV-Vis spectrophotometer (Shimadzu Corporation, Kyoto, Japan) for the assessment of quantity and purity.

\subsection{PCR-DGGE analysis}

The protocols used for PCR amplification and DGGE analysis on the three cheese production replicates are those previously described by Aquilanti et al. (2013). The universal primers U968-f and L1401-r targeting the V6 to V8 region of the 16S rRNA gene, proposed by Randazzo et al. (2002) for the investigation of cheese eubacterial diversity, were used. Both the direct and bulk approach were applied in parallel to the cheese production replicates of each cheese type. Amplification reactions and DGGE runs were performed in duplicate on the same extracts.

The DCode universal mutation detection system (Bio-Rad, Milan, Italy) was used for DGGE analysis of 20-mL aliquots of the PCR products. All the electrophoreses were carried out in $8 \%$ polyacrylamide (acrylamide/bis-acrylamide mix 37.5:1, w/v) gels with a 50.0 to $70.6 \%$ urea-formamide $(w / v)$ gradient $(100 \%$ denaturant was $7 \mathrm{M}$ urea plus $40 \%, w / v$, formamide) increasing in the direction of electrophoresis. Gels were subjected to a constant voltage of $130 \mathrm{~V}$ for $8 \mathrm{~h}$ at $60{ }^{\circ} \mathrm{C}$. The gels were stained in $1.25 \times \mathrm{TAE}$ buffer added with an aqueous ethidium bromide solution, rinsed in distilled water and photographed under UV light with a digital camera. All DGGE bands (labelled with unique and progressive alphanumeric codes) were excised from the gels with sterile pipette tips and transferred into microtubes containing $50 \mu \mathrm{L}$ of TE buffer for elution of the DNA (Garofalo et al. 2008). After overnight incubation at $4{ }^{\circ} \mathrm{C}, 6 \mu \mathrm{L}$ of the DNA suspension was re-amplified with the same primer set (deprived of the GC clamp), purified and sent to Macrogen (Amsterdam, The Netherlands) for sequencing. Identification of the closest known relatives of the 16S rRNA gene sequences was performed through a search in the GenBank DNA database (http://www.ncbi.nlm.nih.gov/) using 
Blast. A sequence identity equal to or higher than $97 \%$ was chosen as a threshold for unambiguous assignation into species.

\section{Results and discussion}

Culturable cell counts are reported in Table 1. With few exceptions, a high variability was seen among the cheeses analysed and especially between raw milk cheeses manufactured with natural starter cultures and pasteurised milk cheeses manufactured with commercial starters. For most cheese types, a variability was even seen among the three cheese production replicates, with differences of up to two orders of magnitude, as in the case for presumptive lactobacilli enumerated in raw milk caciotta cheese ca_r2 (cheese replicates 22, 23 and 24).

With regards to PCR-DGGE results, due to the peculiar characteristics of the $16 \mathrm{~S}$ rRNA, which is recognised by taxonomists as a slowly diverging molecule (Felis and Dellaglio 2007), a few sequences could only be assigned to the Lactobacillus casei group, which includes the closely related species Lactobacillus casei, Lactobacillus rhamnosus, Lactobacillus zeae and Lactobacillus paracasei (Felis and Dellaglio 2007), to the Leuconostoc mesenteroides/pseudomesenteroides group and to the Lactobacillus sakei/curvatus group (Table 2).

Overall, discrepancies were seen when the PCR-DGGE fingerprints obtained with the bulk approach from the three cheese production replicates were comparatively evaluated, irrespective of the cheese variety analysed, in terms of either number of taxa identified or their relative abundance. In Fig. 1, the fingerprints obtained with the bulk approach from the three ca_rl production replicates (coded from 1 to 3 ) are reported as an example; in Table $\overline{\mathrm{S}} 1$, the closest relatives, the percent identities and the accession numbers of the sequences obtained from the DGGE bands shown in Fig. 1 are given. Besides the microorganisms stably identified in this cheese manufacture, such as those ascribed to the Leu. mesenteroides/pseudomesenteroides group and to Str. thermophilus, other microbes were detected in only one (Streptococcus pasteurianus, cheese replicate 1, panels a and d) or two (L. lactis, cheese replicates 2 and 3, panels c, $\mathrm{e}$, f and h; Lact. casei group, cheese replicates 1 and 3, panels c and g) cheese production replicates. Even in terms of relative abundance of the identified microorganisms, discrepancies were observed among cheese production replicates, as for example in the case of $L$. lactis which was detected in the $M 17_{22}$ bulks of cheese replicates 2 (Fig. 1e) and 3 (Fig. 1f) at a level of about 7 and $5 \log$ cfu.g ${ }^{-1}$, respectively.

A neatly higher reproducibility was seen in the fingerprints produced with the direct approach from the cheese replicates of the same cheese manufacture (Fig. 2). Comparable fingerprints were also obtained when replicates of the amplification reactions and DGGE runs were performed on the same extracts, irrespective of the analytical approach used (data not shown). Due to the variability in the fingerprints of the cultivable communities of the cheese production replicates of the same cheese manufacture, one replicate for each cheese manufacture was arbitrarily chosen in order to draw a detailed picture of the composition of the bacterial population revealed by the combined direct and bulk approach (Table 2).

As shown in Table 2, the PCR-DGGE analysis of the DNA extracted directly from cheeses revealed the presence of DNA sequences ascribable to entities closely 


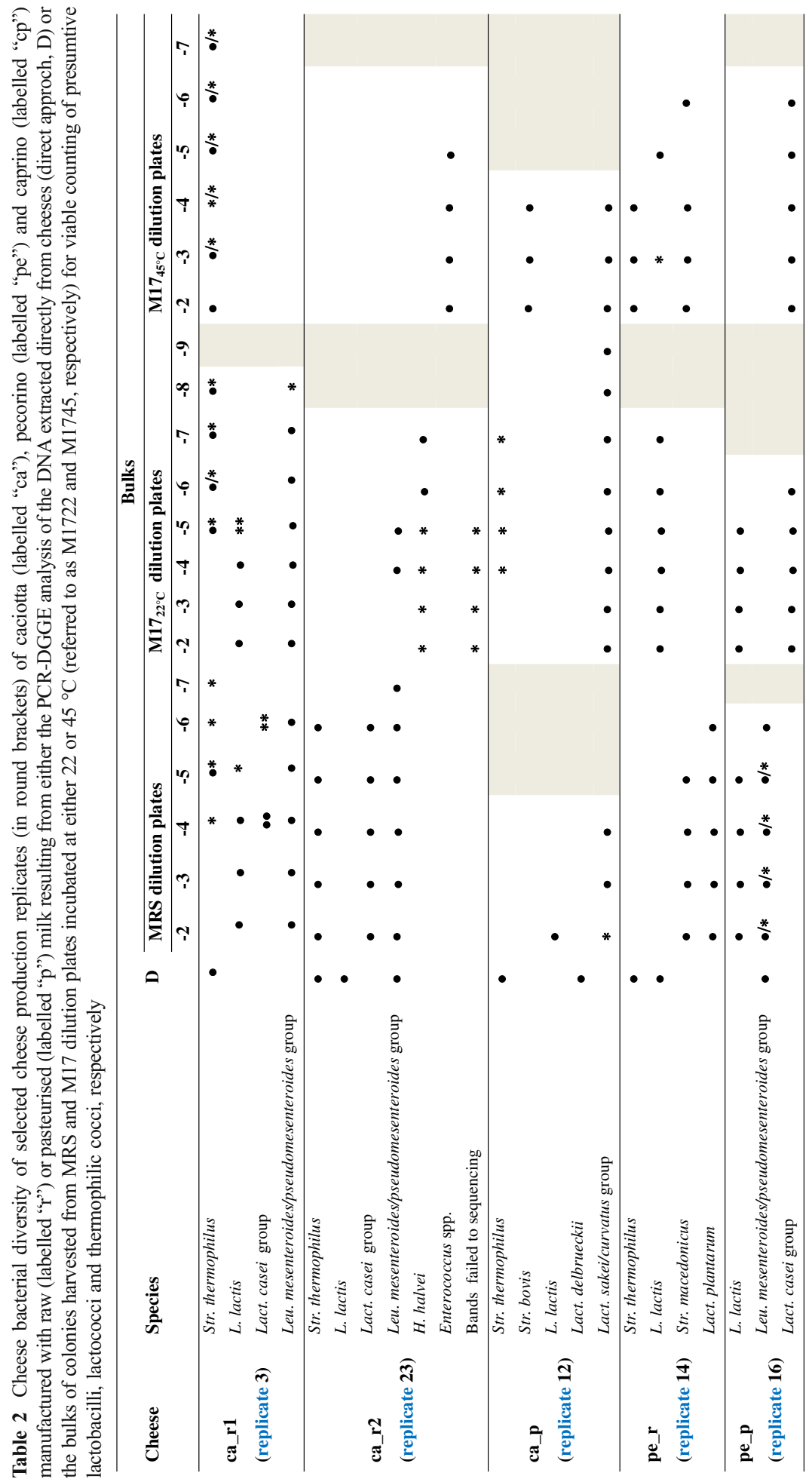

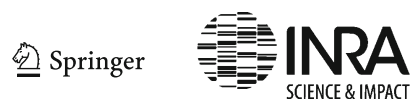




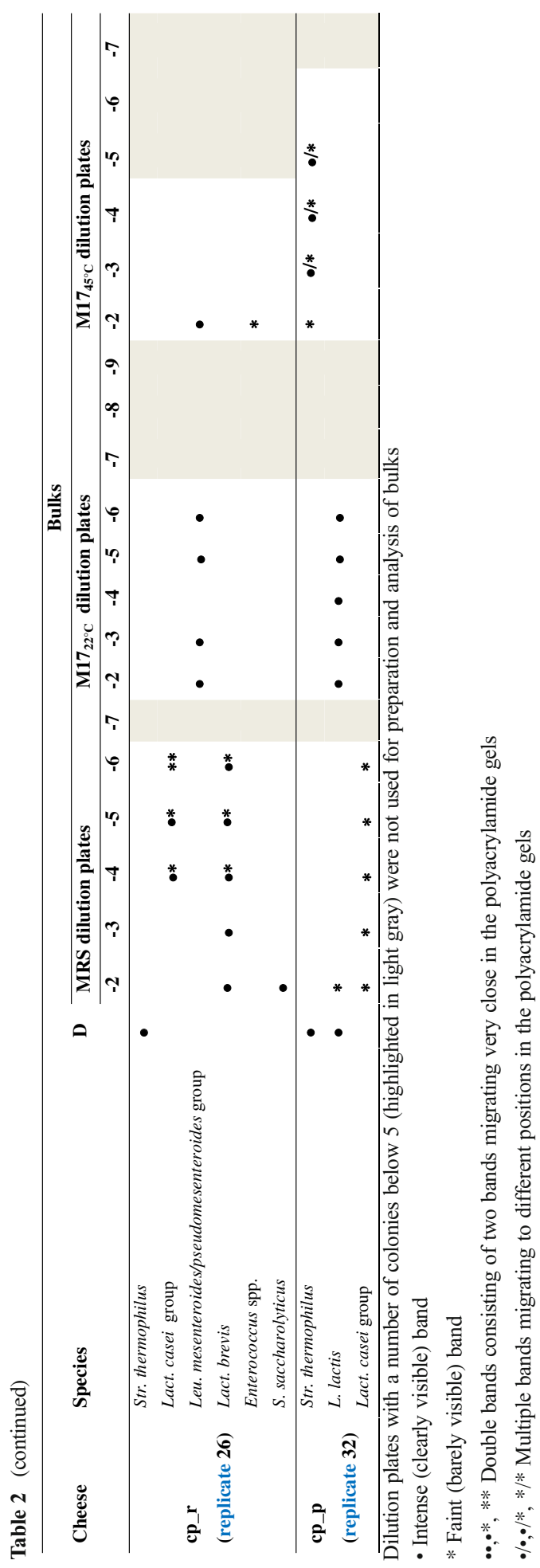




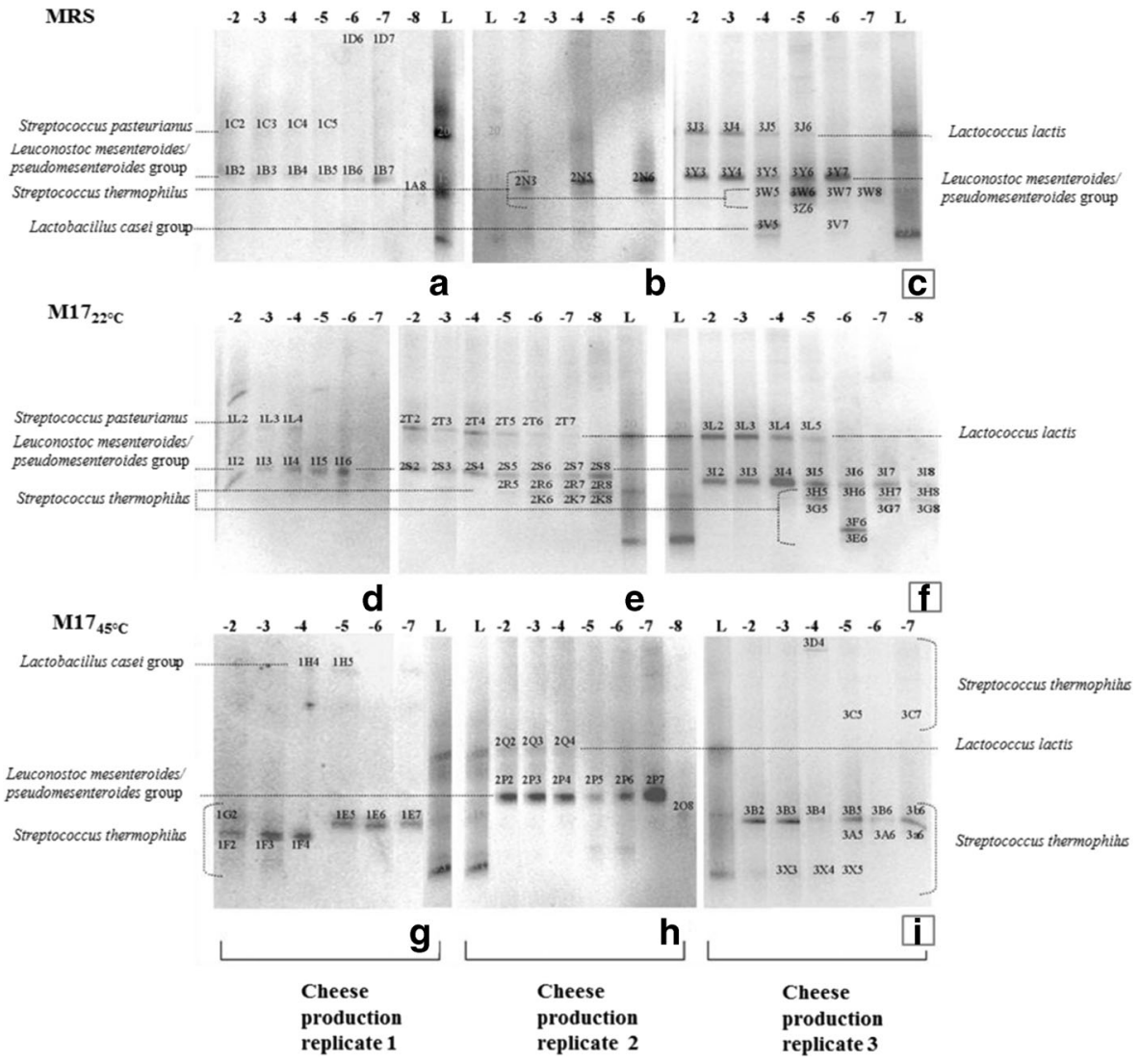

Fig. 1 PCR-DGGE profiles of the three production replicates (coded 1 to 3 ) of raw milk caciotta cheese ca r1 obtained by analysing the bacterial DNA extracted from the bulk cells harvested from MRS (panel a-c) and M17 dilution plates incubated at either $22{ }^{\circ} \mathrm{C}$ (panel $\mathbf{d}-\mathbf{f}$ ) or $45{ }^{\circ} \mathrm{C}$ (panel $\mathbf{g}$-i) for the enumeration of presumptive lactobacilli, lactococci and thermophilic cocci, respectively. Each lane corresponds to a dilution factor or a DGGE ladder $(L)$. Bands subjected to excision and DNA sequencing are labelled with progressive unique alphanumeric codes. Grey boxes identify panels used for mapping the ca_rl bacterial diversity shown in Table 2

related to Str. thermophilus (all the cheeses except pe_p), L. lactis (ca_r2, pe_r and $\mathrm{cp} \_\mathrm{p}$ ), Leu. mesenteroides/pseudomesenteroides group (ca_r2 and pe_p) and Lactobacillus delbrueckii (ca_p).

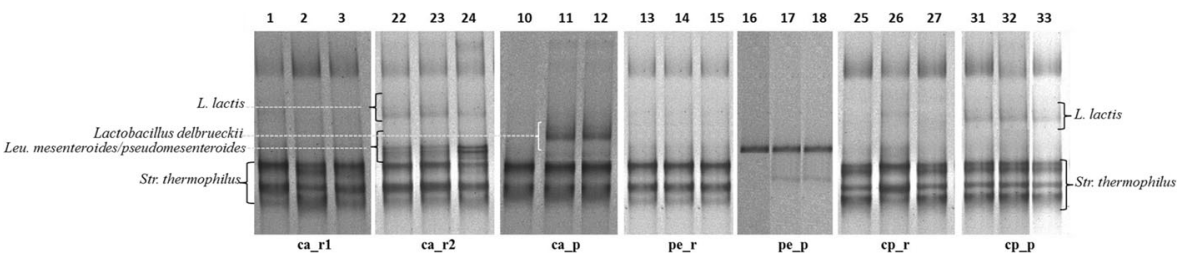

Fig. 2 PCR-DGGE profiles of the three production replicates of raw milk caciotta cheese ca r1 (replicates 1 , 2, 3), ca_r2 (replicates 22, 23, 24), ca_p (replicates 10,11,12), pe_r (replicates 13, 14, 15), pe_p (replicates 16, $17,18)$, cp_r (replicates $25,26,27)$ and $\mathrm{cp} \_\mathrm{p}$ (replicates $31,32,33$ ) obtained by analysing the bacterial DNA extracted directly from the cheese replicates 
A higher number of taxa were identified with the bulk approach (Table 2), and either starter (Str. thermophilus, L. lactis) or non-starter lactic acid bacteria were detected, together with adventitious microorganisms (Streptococcus macedonicus, Staphylococcus saccharolyticus and Streptococcus bovis) and even coliforms (Hafnia alvei). Among non-starter LAB, both hetero- and homo-fermentative taxa were found, including Lactobacillus plantarum, Lact. brevis, Leu. mesenteroides/pseudomesenteroides, Enterococcus spp. and members of the Lact. casei group.

The solid media (MRS and M17) and culturing conditions traditionally recommended for the enumeration of lactobacilli, lactococci and thermophilic cocci including Str. thermophilus showed a very low degree of selectivity, e.g. revealed by the growth of thermophilic streptococci (Str. thermophilus, Str. pasteurianus) on M17 agar incubated at $22{ }^{\circ} \mathrm{C}$ or of mesophilic lactobacilli (Lact. casei group) on M17 agar incubated at 45 ${ }^{\circ} \mathrm{C}$. As dilution factors of bulk DGGE fingerprints were considered, additional information could be gained about the relative abundance of each taxa detected. Overall, leuconostocs were prevailing in half the cheese analyses, being detected up to the highest dilutions (-8) (Table 2). By contrast, the starter mesophilic species L. lactis was preferentially detected at the first and intermediate dilutions (up to -6), whereas thermo-tolerant enterococci were only sporadically identified, preferentially as minority species (as in cp_r and ca_r2).

The first evidence emerging from the overall results of this study is represented by the variability among the cheese replicates manufactured from the same batch of milk and ripened under the same conditions, in terms of both number of taxa identified and their relative abundance. Such a variability emerges even when the culturable cell counts of presumptive lactobacilli, lactococci and thermophilic cocci are comparatively evaluated among cheese replicates from the same cheese production. This finding is in good agreement with what has very recently been found in a research study aimed at monitoring the microbiota involved in Fontina PDO cheese production (Dolci et al. 2014). When drafting the microbial diversity occurring in the Fontina PDO cheeses, the authors considered a species as present if the corresponding band occurred in at least one of the two replicates analysed and as absent if the corresponding band could not be detected in either of the two replicates. Overall, these findings suggest the great impact of the sampling approach on cheese microbial diversity estimation. In this regard, two alternative approaches might allow a more exhaustive profiling of the cheese microbiota to be achieved: the collection and separate analysis of at least two cheese production replicates, in analogy to what was performed in this study, or the collection and analysis of bulk cheese from different replicates. In both cases, an adequate homogenisation of the cheese matrix is required to ensure that certain species do not remain undetected, as recently elucidated by Neviani et al. (2013). For the same purpose, the collection of cheese aliquots from different sections of the cheese, from under the rind to the cheese core, is also recommended in order to identify species which are not homogeneously distributed owing to the different environmental conditions occurring at different depths, as demonstrated in another semi-hard cheese (Dolci et al. 2010).

As the fingerprints obtained by analysing the DNA extracted directly from selected cheese replicates and the bulks were comparatively evaluated, neither the direct nor the bulk approach alone provided such an exhaustive picture of cheese bacterial diversity as the one obtained by the combination of the two techniques. In some cases, such as ca_r1, the analysis of the cultivable community allowed a higher biodiversity to be 
detected, in terms of number of taxa identified, while in others, such as cp_r, species not detected in the bulks were, by contrast, identified through the direct approach. This evidence is substantially in agreement with what was previously highlighted by Ercolini et al. (2001), thus decisively confirming the importance of combining the sequencing of DGGE bands after direct DNA extraction with the cultivation of microorganisms.

A wide range of hypotheses have been put forward by various authors to explain analogous observations, such as the amplification, with the direct approach, of nucleic acids extracted from dead but intact cells or stressed/injured cells unable to grow on the selective media used, the occurrence of differences in the detectability threshold of different targeted species and the masking effect of the most abundant templates during PCR.

The hypothesis of a competition among templates during the amplification reaction is clearly supported by the fingerprints of the M17 22 and $M 17_{45}$ bulks referred to as ca_r2 and pe_r, respectively. More specifically, for ca_r2, an increase in the intensity of the band closely related to $H$. alvei was seen at dilution -6 , when multiple bands (ascribed to the Leu. mesenteroides/pseudomesenteroides group or which failed sequencing) detected up to dilution -5 disappeared. Analogously, for pe_r, a faint band closely related to L. lactis was barely detectable at dilution -3, when DGGE fingerprints were dominated by intense bands closely related to Str. macedonicus and Str. thermophilus; interestingly, at dilution -5 , the band closely related to L. lactis became more visible, whereas the one closely related to Str. macedonicus and Str. thermophilus conversely disappeared. Finally, at dilution -6 , the band ascribable to L. lactis again disappeared, whereas the one ascribable to Str. macedonicus once again became clearly detectable. In this latter case, the preferential amplification of L. lactis or Str. macedonicus target sequences was apparently not dependent on their relative abundance, but seems more likely to be the result of stochastic, and hence unpredictable, fluctuations in the number of amplicons produced during the PCR reaction.

An unpredictable competition among templates is also supported by the detection of a few species at the highest but not the lowest dilutions, as in the case of Str. thermophilus identified in both the MRS and M17 22 bulks of ca_r1 from dilutions -4 and -5 up to -7 and -8 , respectively. This finding clearly suggests the importance of increasing as far as possible the number of dilution factors to be analysed once the PCR-DGGE bulk approach is applied to the investigation of food bacterial communities, in contrast with what is routinely performed by food microbiologists, who generally limit their analyses to selected plates, often the countable ones.

When the results of the bulk analysis are carefully evaluated, a range of further evidence emerges, including the poor selectivity of the growth media and incubation conditions used and traditionally recommended for counting and isolation of lactobacilli, lactococci and thermophilic cocci, respectively. In some cases, colonies of the thermophilic species Str. thermophilus grew on M17 agar plates incubated at $22^{\circ} \mathrm{C}$ but not at $45^{\circ} \mathrm{C}$ (ca_p), whereas the mesophilic species $L$. lactis, known to clearly prefer incubation temperatures around $30^{\circ} \mathrm{C}$, was stably detected in the M17 bulks harvested from plates incubated at $45^{\circ}$ (pe_r) or even in the MRS bulks (ca_r1, pe_p, cp_p and ca_p). The latter is the medium of choice for the enumeration and isolation of lactobacilli, and the findings are also in agreement with those previously reported (Ercolini et al. 2001). On the one hand, this evidence encourages ongoing research efforts to develop new selective media for the 
isolation and enumeration of starter and non-starter LAB from cheese and dairy products, while on the other, it also clearly demonstrates the need to use a series of more selective media for the analysis of the cultivable fraction of cheese bacterial communities by PCRDGGE, including acidified MRS (pH 5.7), whey agar medium (WAM), cheese agar medium (CAM), etc. (Neviani et al. 2013).

Further non-negligible evidence using this approach is the identification of the dominating taxa, in agreement with what was previously found by Ercolini et al. (2001). Indeed, the results collected from the cheeses analysed in the present study with the bulk approach allowed the concentration of the single species found in the DGGE bulk profiles to be roughly ascertained. By way of an example, the bacterial community of ca $\mathrm{p}$ was apparently dominated by members of the Lb. sakei/curvatus group, which were stably detected up to the highest dilutions in the M17 22 bulks, and to a lesser extent by thermo-tolerant streptococci (detected up to the dilutions -7 and -4 , respectively) and mesophilic lactococci (detected at the sole dilution -2 ).

Due to the high potential of the bulk approach, which involves the analysis of the whole set of dilution plates used for bacterial enumeration, Ercolini (2004) proposed the sequencing of the DGGE bands visualised at the highest dilutions as an alternative to the isolation of pure cultures. However, on the basis of the overall results collected in this study, such a choice might lead to an underestimation of the real bacterial diversity of cheeses and dairy products, mainly due to the loss of information about minority species, which by contrast are preferentially revealed by the sequencing of the DGGE bands visualised at the lowest dilutions.

Based on the fingerprints arising from the PCR-DGGE analysis of the bulk suspensions, even the use of single DGGE bands as biodiversity markers for the calculation of simple mathematical indexes, which to date has been exploited for the differentiation of dairy products, might be carefully considered case by case. In fact, in some cases, multiple bands ascribed to the same species were visualised, feasibly due to the occurrence of multiple heterogeneous copies of the 16S rRNA gene. This evidence, which was previously pointed out by other authors investigating cheese microbiota (Ercolini 2004; Ricciardi et al. 2014), makes the sequencing of all the visualised DGGE bands a pre-requisite for the assessment of the degree of cheese microbial complexity for each bulk analysed through the calculation of biodiversity indexes.

Finally, further interesting evidence emerged from the comparison of the culturable cell counts and the DGGE fingerprints of the bulks. In most cases, there was a concordance between the relative abundance of presumptive lactobacilli, lactococci and thermophilic cocci and the composition of the bacterial community revealed by PCR-DGGE. However, some discrepancies were found when culturable cell counts were compared with the relative abundance of some identified species, especially as regards thermophilic cocci. The latter finding was particularly evident in $\mathrm{cp}$ _r, where a population of presumptive thermophilic cocci higher than $4 \log \mathrm{cfu}^{-1} \mathrm{~g}^{-1}$ was counted, but no thermophilic or relatively thermotolerant microorganisms, such as those belonging to the genus Enterococcus, were detected at levels higher than $2 \log$ cfu.g ${ }^{-1}$ by DGGE analysis of the $M 17_{45}$ bulks. Once again, this finding might be ascribed to the low selectivity of the growth media used or even to the biases of PCR-DGGE detailed above.

As far as the bacterial diversity of the raw and pasteurised cheeses analysed is concerned, no clear discrepancies in the occurrence of starter and adventitious LAB species were seen. This finding agrees well with those emerged in a previous research study focussed on the 
characterisation of analogous semi-hard Italian cheeses (Aquilanti et al. 2013). This can be explained by both the starter lactic acid bacteria capability of surviving pasteurisation or gaining entry to the milk after pasteurisation at any stage of the cheese-making process and to the high adaptation of starter species, such as Str. thermophilus and L. lactis, to the cheese environment, irrespective of their deliberate use in cheese-making.

\section{Conclusions}

The novelty of the present study is undoubtedly represented by the objective analysis of the weaknesses and strengths of a poorly explored combined PCR-DGGE approach for the semi-quantitative characterisation of bacterial cheese communities. The results overall collected clearly highlighted the usefulness of a combination of the direct and bulk PCRDGGE approaches for the investigation of cheese bacterial diversity rather than the sole analysis of the nucleic acids extracted directly from cheeses, which currently represents the standard procedure in PCR-DGGE-based cheese ecological investigations. Regarding the bulk approach, the use of multiple solid media might effectively contribute to a better profile of the cultivable fraction of the cheese bacterial communities. Even the choice of the cheese sampling strategy and the dilution factors to be considered for the preparation of bulks was found to be relevant. Regarding dilution factors, the analysis of colonies collected in bulk from all the MRS and M17 dilution plates incubated at different temperatures allowed either the identification of both dominant and minority species or their quantification. Finally, the bulk approach proposed was also found to markedly reduce the impact of technical biases, such as the competition among the most abundant templates or the stochastic fluctuations in the amplification efficiency of different templates, on the profiling of cheese cultivable communities. However, the bulk analysis undoubtedly presents two main drawbacks: it is particularly laborious and, consequently, time-consuming; moreover, the analysis of a high number of bulks from all the dilution plates of different solid media implies the processing of numerous PCR samples and hence of polyacrylamide gel runs. A valid compromise between the analysis of the whole set of dilution plates and the analysis of the sole plates showing a colony number comprising between 30 and 300 might be represented by the analysis of at least two dilution plates preferentially selected among the highest and the lowest dilutions, in order to have a more complete picture of both the dominant and minority species, respectively.

Acknowledgments This work was supported by a grant (Rule 37/99) from the Marche Region, Italy.

\section{References}

Aquilanti L, Santarelli S, Babini V, Osimani A, Clementi F (2013) Quality evaluation and discrimination of semi-hard and hard cheeses from the Marche region (Central Italy) using chemometric tools. Int Dairy J 29:42-52

Delgado S, Rachid CT, Fernández E, Rychlik T, Alegría A, Peixoto RS, Mayo B (2013) Diversity of thermophilic bacteria in raw, pasteurized and selectively-cultured milk, as assessed by culturing, PCRDGGE and pyrosequencing. Food Microbiol 36:103-111

Dolci P, Alessandria V, Rantsiou K, Bertolino M, Cocolin L (2010) Microbial diversity, dynamics and activity throughout manufacturing and ripening of Castelmagno PDO cheese. Int J Food Microbiol 143:71-75 
Dolci P, De Filippis F, La Storia A, Ercolini D, Cocolin L (2014) rRNA-based monitoring of the microbiota involved in Fontina PDO cheese production in relation to different stages of cow lactation. Int $\mathrm{J}$ Food Microbiol 185:127-135

Ercolini D (2004) PCR-DGGE fingerprinting: novel strategies for detection of microbes in food. J Microbiol Methods 56:297-314

Ercolini D, Moschetti G, Blaiotta G, Coppola S (2001) The potential of a polyphasic PCR-DGGE approach in evaluating microbial diversity of natural whey cultures for water-buffalo Mozzarella cheese production: bias of culture-dependent and culture-independent analyses. Syst Appl Microbiol 24:610-617

Ercolini D, Hill PJ, Dodd CER (2003) Bacterial community structure and location in Stilton cheese. Appl Environ Microbiol 69:3540-3548

Ercolini D, Mauriello G, Blaiotta G, Moschetti G, Coppola S (2004) PCR-DGGE fingerprints of microbial succession during a manufacture of traditional water buffalo mozzarella cheese. J Appl Microbiol 96:263270

Felis G, Dellaglio F (2007) Taxonomy of lactobacilli and bifidobacteria. Curr Issues Intest Microbiol 8:44-61

Garofalo C, Silvestri G, Aquilanti L, Clementi F (2008) PCR-DGGE analysis of lactic acid bacteria and yeast dynamics during the production processes of three varieties of Panettone. J Appl Microbiol 105:243-254

Garofalo C, Osimani A, Milanović V, Aquilanti L, De Filippis F, Stellato G, Di Mauro S, Turchetti B, Buzzini P, Ercolini D, Clementi F (2015) Bacteria and yeast microbiota in milk kefir grains from different Italian regions. Food Microbiol 49:123-133

Mayo B, Rachid CT, Alegría A, Leite AM, Peixoto RS, Delgado S (2014) Impact of next generation sequencing techniques in food microbiology. Curr Genomics 15:293-309

Neviani E, Bottari B, Lazzi C, Gatti M (2013) New developments in the study of the microbiota of raw-milk, long-ripened cheeses by molecular methods: the case of Grana Padano and Parmigiano Reggiano. Front Microbiol 4:1-14

Osimani A, Garofalo C, Aquilanti L, Milanović V, Clementi F (2015) Unpasteurized commercial boza as a source of microbial diversity. Int J Food Microbiol 194:62-70

Pogačić T, D'Andrea M, Kagkli DM, Corich V, Giacomini A, Baldan E, Majhenič AČ, Obermajer T, Rogelj I, Samaržija D (2011) Biodiversity of microbial consortia isolated from traditional fresh sheep cheese Karakacanski skakutanac. Mljekarstvo 61:208-209

Quigley L, O'Sullivan O, Beresford TP, Ross RP, Fitzgerald GF, Cotter PD (2011) Molecular approaches to analysing the microbial composition of raw milk and raw milk cheese. Int J Food Microbiol 150:81-94

Randazzo CL, Torriani S, Akkermans ADL, de Vos WM, Vaughan EE (2002) Diversity, dynamics and activity of bacterial communities during production of an artisanal Sicilian cheese as evaluated by 16S rRNA analysis. Appl Environ Microbiol 68:1882-1892

Rantsiou K, Urso R, Dolci P, Comi G, Cocolin L (2008) Microflora of Feta cheese from four Greek manufacturers. Int J Food Microbiol 126:36-42

Ricciardi A, Blaiotta G, Di Cerbo A, Succi M, Aponte M (2014) Behaviour of lactic acid bacteria populations in Pecorino di Carmasciano cheese samples submitted to environmental conditions prevailing in the gastrointestinal tract: evaluation by means of a polyphasic approach. Int J Food Microbiol 179:64-71

Van Hoorde K, Verstraete T, Vandamme P, Huys G (2008) Diversity of lactic acid bacteria in two Flemish artisan raw milk Gouda-type cheeses. Food Microbiol 25:929-935

Van Hoorde K, Heyndrickx M, Vandamme P, Huys G (2010) Influence of pasteurization, brining conditions and production environment on the microbiota of artisan Gouda-type cheeses. Food Microbiol 27:425433 